SUPPLEMENT

\title{
Heading in football. Part 2: Biomechanics of ball heading and head response
}

\author{
N Shewchenko, C Withnall, M Keown, R Gittens, J Dvorak
}

Br J Sports Med 2005;39(Suppl I):i26-i32. doi: 10.1136/bjsm.2005.019042

See end of article for authors' affiliations

.....................

Correspondence to: N Shewchenko, Biokinetics and Associates Ltd, Ontario, Canada; shewchenko@biokinetics. com

\begin{abstract}
Objectives: Controversy surrounding the long term effects of repeated impacts from heading has raised awareness among the public and the medical community. However, there is little information about the human response to the impacts and what measures can be taken to alter their effect. The objective of the current study was to gain a better understanding of heading biomechanics through the implementation of a numerical model and subsequent investigation of parameters related to heading technique and ball characteristics.

Methods: A controlled laboratory study was carried out with seven active football players, aged 20-23 years who underwent medical screening and were instrumented with accelerometers mounted in bite plates and electromyographic electrodes on the major neck muscle groups. Balls were delivered at two speeds $(6 \mathrm{~m} / \mathrm{s}$ and $8 \mathrm{~m} / \mathrm{s})$ as the subjects demonstrated several specific heading manoeuvres. Photographic targets were tracked via high speed video to measure heading kinematics. One subject demonstrating reasonably averaged flexion-extension muscle activity phased with head acceleration data and upper torso kinematics was used to validate a biofidelic 50th percentile human numerical model with detailed representation of the head and neck.

Results: Heading kinematics and subject responses were used with a detailed numerical model to simulate impact biomechanics for a baseline heading scenario. Changes to heading techniques and ball characteristics which mitigated head impact response were identified.

Conclusion: A numerical model combined with biomechanical measurement techniques is an important tool for parametric investigation of strategies to reduce head impact severity via changes in heading technique or the physical properties of the ball.
\end{abstract}

$\mathrm{N}$ umerical models have been used by researchers to gain a better understanding of football heading biomechanics and methods for reducing head impact response. ${ }^{1-4}$ The advantages of numerical models include repeatability, ease of altering and controlling specific model characteristics, and ability to acquire detailed response information. Recognised disadvantages include the need for validation of the models for conditions under which the model is exercised.

An early modelling effort consisted of a rigid body representing the head, seven bodies for the neck, and one for the torso. ${ }^{1}$ Springs and dampers interconnected the bodies to represent the overall interactions of the discs, ligaments, and muscles. The model was validated for passive football impact conditions represented by force-time functions and with experimental data. The effects of head/ball mass ratio, impact velocity, and elasticity were investigated to propose safe playing conditions. Linear and angular acceleration injury criteria from automotive safety research were employed to assess the risk of acute brain injury.

A numerical model employed by Ziejewski et al consisted of three dimensional rigid bodies implemented in the Articulate Total Body (ATB) computer simulation software. ${ }^{2}$ Normally consisting of 15 segments, this model was reduced to only three bodies: a head, a neck, and a torso. The neck was represented by a single body with upper and lower neck pivots. A ball and socket joint attached the head to the upper pivot and a more complex ball-socket and slip joint with a spring damper connected the lower pivot to the torso. The model was validated to reproduce the passive fore-aft acceleration response of the 50th percentile Hybrid III automotive test dummy head-neck structure subjected to freefall football impacts with a padded headband. In the model and validation, the neck was connected to a lump mass and test table representing the torso. Motion of the head approaching the ball during a header was not considered. The modelling efforts were continued with rigid bodies and finite element methods to study the efficacy of reducing head trauma during heading with headbands. ${ }^{3}$ Peak linear and angular acceleration, the head injury criterion, and cumulative strain damage measure were used to assess acute brain injury risk.

In another application, the principles of classic elastic theory were employed in an analytical model to study ball to head impacts for different aged children and variations in ball pressure and size. ${ }^{4}$ For the contact stress distribution model to work, both the player head and ball were considered linearly elastic and the contact surface between them was assumed to be flat. This study found that the ball pressure had little or no effect on the head response, which included peak impact force and linear head acceleration. The ball stiffness parameter in the model was determined from physical compression tests of balls at $0.5 \mathrm{~m} / \mathrm{s}$, which is considerably slower than the impact velocities observed in the current study (up to $11 \mathrm{~m} / \mathrm{s}$ ).

Although the above models have enhanced our understanding of head impacts in football, improvements are required to study possible countermeasures for reducing impact severity including modified heading techniques and the impact environment. The ability to investigate the effects of neck muscle activation, body segment alignment, directional sensitivity, and ball characteristics was required for the current study.

An early version of a head and neck model implemented in MADYMO software was used to study the effect of headneck coupling on head response experienced during head to head impacts in the American National Football League. ${ }^{5}$ The 
software code modelled the kinematics and kinetics of deformable bodies, constrained joints, and internal forces from joint torques or muscle models. In the current implementation, the cervical and first thoracic vertebrae of this model provided sufficient neck segmentation to simulate observed head-neck kinematics. In addition, the head and neck model had been validated with human subject data in several directions including frontal, lateral, and posterior. ${ }^{6}$ Unfortunately, this version of the model was not integrated onto a body and, having only passive neck muscles it was not possible to represent the muscle activations and head-necktorso coupling required for studying heading techniques.

In another study an updated MADYMO head and neck model was used to investigate the neck response under low severity automotive type impacts. ${ }^{7}$ The head and neck model, which now included active muscle elements, was integrated into the body of a 50th percentile male model. The combined model had previously been validated against typical automotive-type responses but was now subjected to rearward impacts to the chin and forehead and vertical loading to the chin. Measurements during human subject testing provided input for impact simulations as well as for validation data. Muscle activation levels available in the model were exercised to match head and neck kinematics in the subject video. The results showed that the neck model was suitable for predicting head and neck kinematics and the model head acceleration correlated well with output from accelerometers mounted to subjects' heads.

The modelling approach for the current study required accurate representation of the head, neck structure, and torso in terms of their mass properties. Realistic head to ball impact compliance, active and passive neck musculature and a means to reproduce upper body heading motion were essential requirements that dictated the selection of the model. These requirements led to the selection of the validated human model with detailed head and neck implemented in MADYMO. The objectives of this study were to:

- develop a numerical model for the study of biomechanical heading parameters

- determine the effects of heading techniques on head impact response.

The study comprises the second part of a three part series. Part 1 of this series "Development of biomechanical methods to investigate head response" deals with the development of biomechanical measurement methodology and human subject trial results. ${ }^{8}$ A complementary study of external factors influencing head response-that is, ball impacts, is presented in Part 3 "Effect of ball properties on head response". 9 The numerical model described in this paper was used for Part 3.

\section{METHODS}

In this study we combined the measured kinematics and kinetics of human test subjects with numerical modelling to gather rudimentary information on heading biomechanics. The numerical model allowed us to explore many parameters that would otherwise be difficult to achieve and control with humans. In addition, variability of the results was eliminated thereby providing clear insight into the effects of each parameter. Thus we could investigate the effect of different heading techniques and ball characteristics and their implication on head impact severity independently. An overlying assumption was that the change in one or more parameters would not result in changes to the rebound characteristics of the heading manoeuvre. Adaptation or alteration of the remaining model characteristics would therefore not be required.
Implementation of the model was based on the kinematic and neck muscle EMG data from an initial set of subject trials $^{8}(n=7)$. We selected a single heading scenario (test code: LS2; see table 1, reference 8 for explanation of heading scenario codes) and representative test subject as a baseline for validation and implementation of the model since the kinematic and kinetic responses are dependent on an individual's technique and physical characteristics. With this approach, we could investigate relative changes in heading technique and response and relate back to the response envelope of the subjects.

\section{Model of the athlete}

A 50th percentile male human model with detailed neck (version.1.2.1.1, MADYMO 6.0.1 (Mathematical Dynamic Modeler. (MADYMO), TNO, The Netherlands)) was used to represent a football player. A typical pre-impact configuration is depicted in fig 1. The original human model was validated under frontal, rear, and lateral loading conditions, but it has not been fully validated for loading in the axial direction. Further, previous validations did not involve active use of the model. For the specific impact conditions during heading, additional validation efforts were therefore made.

The passive biofidelity of the head-neck model allowed for certain neck muscle elements to react to inertial and impact loads of the head. Unlike the typical passive use of the model in impact biomechanics, in the current study we used the model actively by forcing the muscle elements to control the motions of the head and neck system, and hence, the rebound kinematics of the ball. With correct representation of the head mass and neck constraints, the computed head and neck responses from the simulated heading scenarios could be related to some measure of impact severity or injury potential.

Proper implementation of the model would result in dynamic similitude between the model and subject. We accomplished this by replicating the kinematics of the player throughout the impact event including correct torso, neck, head, and ball motions just prior to ball impact and for a short time thereafter. Replicating the entire event from subject standstill to full follow through was neither required nor practical due to the short duration of the contact event, significant demands for computational time, and difficulty in configuring the model for extensive pre-impact motion and post-impact response. A simulated time period of $100 \mathrm{~ms}$ was selected to represent adequately the impact event of interest.

To employ the human model, the lumbar and thoracic vertebrae were made rigid, preventing translation and rotation between any two vertebrae. This simplification eased

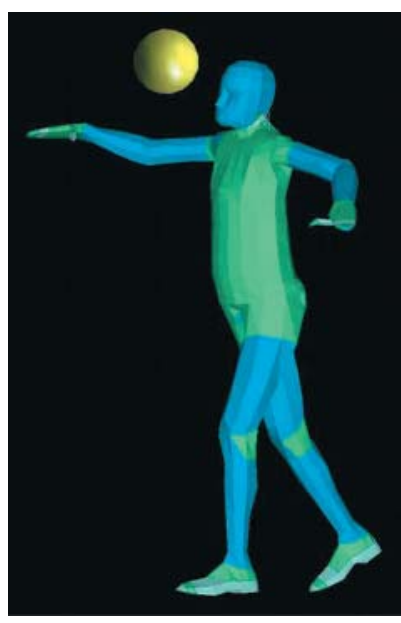

Figure 1 Human model with detailed head and neck. 

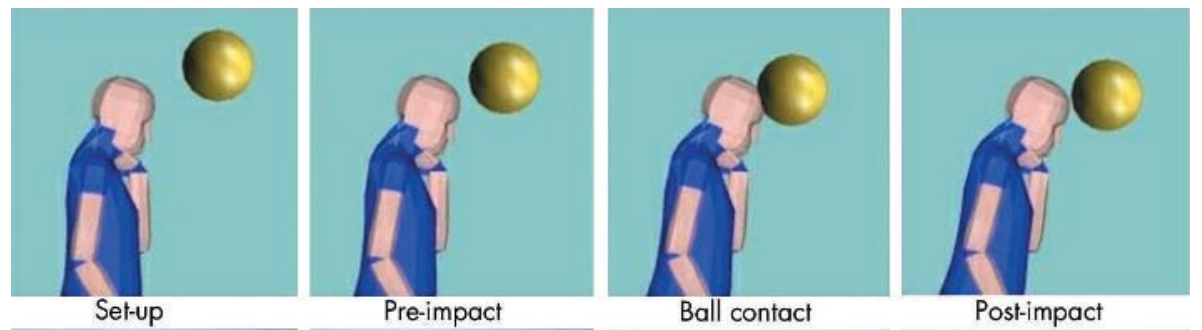

Figure 2 Qualitative comparison of model and subject kinematics.
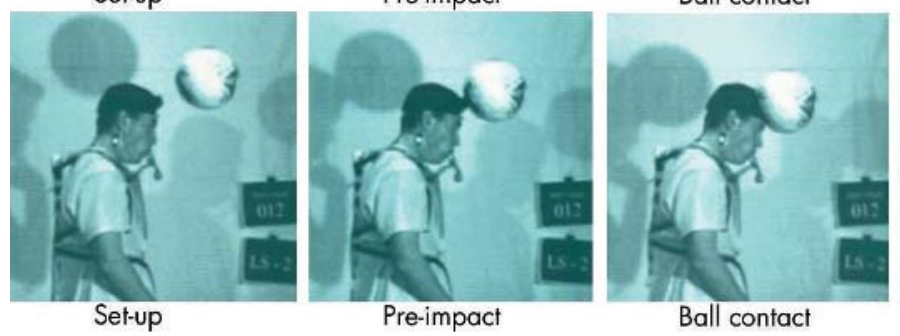

Ball contact

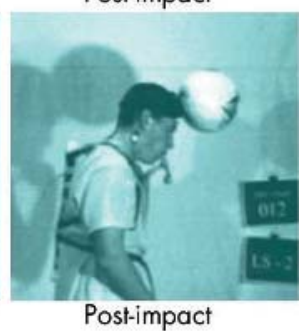

the manipulation of the torso and was justified by the locked torso shape observed in the subject tests. ${ }^{8}$ Position and movement of the torso of the model replicated the kinematics of the representative subject (fig 2). We also added several modelling elements to output specific data such as the location of anatomical landmarks and the angle of the head compared with the volunteer.

\section{Model of the football}

We created the football using a multi-body model with a spherical outer surface. Although the shape or contact area would not affect the interaction between the head and ball for this type of model, the additional fidelity obtained with the use of a more complex model-for example, finite elements-was not warranted since the contact characteristics were based on physical measurement of the ball impact. The simplification was also justified because the modelling efforts were representative of a single player, and it was the relative change in head and neck response for various biomechanical parameters that was of interest. A basic assumption was that strategies benefiting one player would be beneficial to all players.

Our football model was based on the adidas Fevernova TriLance, size 5 ball (adidas, Germany) with a pressure of $80 \mathrm{kPa}$. Properties such as the size and mass of the ball were measured directly. The force-deflection characteristics were measured by impacting the ball onto a steel hemispherical anvil $(\mathrm{r}=98 \mathrm{~mm})$ approximating the contact area and shape of the forehead. The impact force was measured with a piezoelectric force transducer (Endevco Model 2106E; Endevco Corp., San Juan Capistrano, CA) and the ball deflection was measured from high speed video (Redlake Motionscope 1000 set at 500 fps; Redlake, USA). Impact speeds of $7 \mathrm{~m} / \mathrm{s}$ were used to approximate the average ball test speed of the human trials. Ball energy loss or hysteresis was observed to be minimal. A linear approximation of ball stiffness was therefore used to represent both the loading and unloading phases of the ball.

\section{Torso motion}

An initial rotational velocity about the hip joint represented the observed torso motion. This was based on the average velocity prior to impact measured from the subject kinematics. During rotation, the torso can be affected by external forces from ball contact and therefore provides an appropriate effective mass and constraint at the base of the neck. The large mass difference between the ball and the human model resulted in minimal energy transfer to the torso resulting in realistic follow through after impact.

\section{Ball speed}

We measured the ball speed from the subject video over the course of the impact event. An average incoming speed towards the head was computed and used for the ball model. Similarly, average outgoing speed from the head was determined and used for validation purposes. Since the position and speed were recorded as the ball approached the head, the effect of gravity was already taken into account and not included in the simulation.

\section{Muscle activation}

The detailed neck model contained 68 pairs of muscles organised into three groups based on their primary contribution to head and neck movement. The defined groups were named flexor, extensor, and sterno and each group was subdivided into left and right sides (fig 3). The manipulation of each muscle group through activation time histories can result in many common head movements. Since muscles within a given group may have different absolute forces, relative muscle activation was provided as a percentage of the maximal force and each muscle within the group would be activated accordingly. The activation percentage for the model is relative to maximal voluntary contraction (MVC) effort for an average human. The intent was to increase the levels above a baseline value and not necessarily replicate actual levels seen in game play. The dynamic activation levels in the subjects were higher than the measured static MVC levels. The active muscles are controlled by the input parameters, whereas the passive resistance is obtained

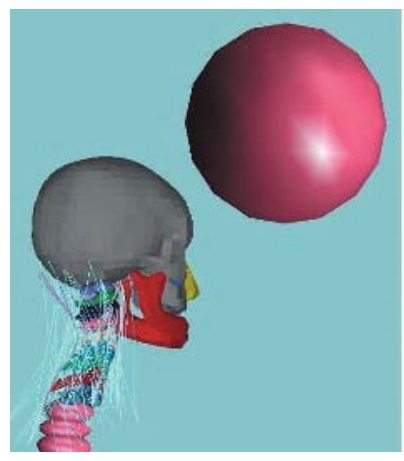

Figure 3 Detailed head and neck model. 
Table 1 Parameter matrix for numerical simulations

\begin{tabular}{lccc}
\hline & \multicolumn{3}{c}{ Muscle group activity level (\%) } \\
\cline { 2 - 4 } Parameter variation & Flexor & Extension & Sterno \\
\hline Baseline & 80 & 0 & 0 \\
Muscle activation & 125 & 10 & 0 \\
Muscle activation & 150 & 15 & 20 \\
Head/torso alignment & 80 & 0 & 0 \\
\hline
\end{tabular}

automatically from reactions to elongation from external loads according to the Hill muscle model.

Muscle activity of the sternocleidomastoid and trapezius muscle groups, measured with electromyography was used to time the activation of the available muscle groupings in the model. But we could establish the required level of muscle force only through model iterations. Because of the initial forward velocity of the model, the activation of the flexor muscles was of particular importance. This muscle group, applied equally to the left and right sides of the neck, was activated to flex the neck and head towards the ball prior to impact. By comparing the head angle of the model with the kinematics of the subject, activation of the remaining muscle groups was adjusted until suitable head orientation at the instant of ball impact was achieved.

\section{Parametric study}

We conducted a parameter study with the numerical model to investigate the effects of muscle activation and torso alignment on head response. The effects were evaluated by comparing the peak head linear and rotational accelerations, upper neck (OC-C1) peak forces, neck moments, and the maximum Head Impact Power (HIP) index ${ }^{10}$ for the model parameters. Additional model outputs include head and torso angles, ball rebound velocity, and torso acceleration at the $\mathrm{Tl}$ vertebra. All variations of the numerical model were compared with the baseline low speed heading configuration (scenario LS2, subject HS12; see table 1, reference 8 for explanation of heading scenarios).

The neck muscle activation levels were modified to evaluate the effects of increased coupling between the head and torso without modifying the overall pre-impact heading kinematics. The baseline set-up was modified by first increasing the activation levels of the flexor muscle groups to a predefined level. Then we performed the simulation and re-examined the kinematics of the head and ball. Through several iterations, the activation levels of the extensor and sterno muscle groups of the model were increased until the resulting kinematics were reasonably similar to the baseline test. This process was completed for two cases where the flexor muscle activation levels were higher than the baseline heading scenario.

The baseline test position of the numerical model was similar to the test subject (scenario LS2) and later modified to evaluate the effects of body alignment. An attempt was made to align the cervical spine and torso with the impact point on the head to provide a more rigid support structure for the head. In the aligned case, the head angle was unchanged from the baseline case $\left(28^{\circ} v 27^{\circ}\right)$ and the torso was inclined in a more horizontal orientation with an angle of $20^{\circ}$ compared with $6^{\circ}$ forwards from vertical. The torso kinematics were based on several iterations that would result in the head passing through the required position and at the right angle at the time of ball impact.

The parameters selected for the numerical simulations are presented in table 1 . In all cases, the adidas Fevernova

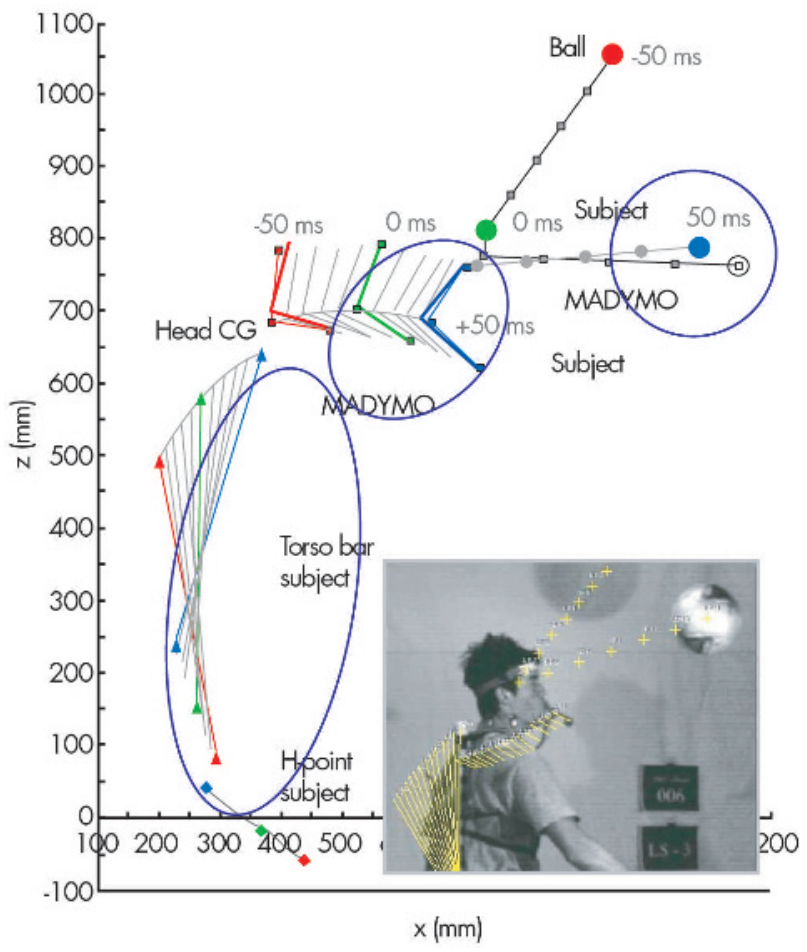

Figure 4 Comparison of model and subject markers.

Tri-Lance ball model was used (pressure $=0.8$ bar, mass $=0.444 \mathrm{~kg}$ dry, dynamic stiffness $=33.6 \mathrm{kN} / \mathrm{m}$, radius $=0.110 \mathrm{~m})$.

\section{RESULTS}

\section{Head kinematic validation}

High speed video recordings of a subject heading the ball provided detailed trajectories of the ball, torso, and head. A comparison between the model and experimental trial for the body and ball kinematics in the low speed baseline heading scenario (LS2) is depicted in fig 2. Qualitatively, it is shown that a good match was obtained before, during, and after impact with the ball.

A quantitative comparison of kinematics was obtained from measurements of the torso and head kinematics with corresponding data from the simulation. For the subject trials, body motion was measured from photographic targets mounted on the torso and head. Similarly, reference landmarks were used to provide translations and rotations of the model. Other data such as the head angle, ball position, and ball speed were also determined. Comparison of these points at discrete time intervals of $10 \mathrm{~ms}$ is shown in fig 4. Torso motion of the model was prescribed by subject data so a perfect match was observed. The kinematics of the model head was a result of the initial conditions and muscle activations. Minor deviations from the subject are observed. The ball trajectory deviations were larger as these depend on the summation of the head motions, contact characteristics, and ball rebound properties. Comparisons of the head translational and rotational kinematic time histories for the head are presented in fig 5. Discrepancies of less than 3\% were observed for head translations at the point of impact while larger discrepancies of $13 \%$ were observed for head rotations.

Validation of the simulation under dynamic conditions was carried out by a comparison of head translational velocity obtained from mathematical differentiation of the kinematics. With comparable velocities, it would be assured that 

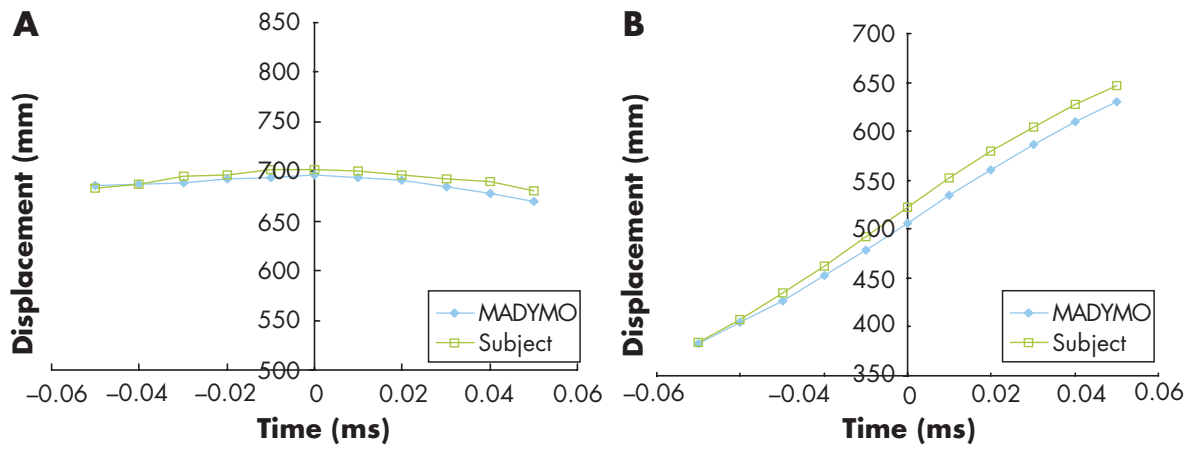

Figure 5 Comparison of subject and model results: (A) vertical translation, (B) horizontal translation, (C) head rotation, and (D) head fore-aft velocity.

C

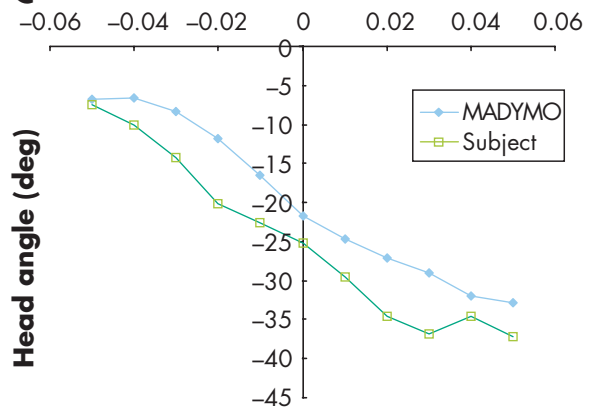

Time (ms)
D

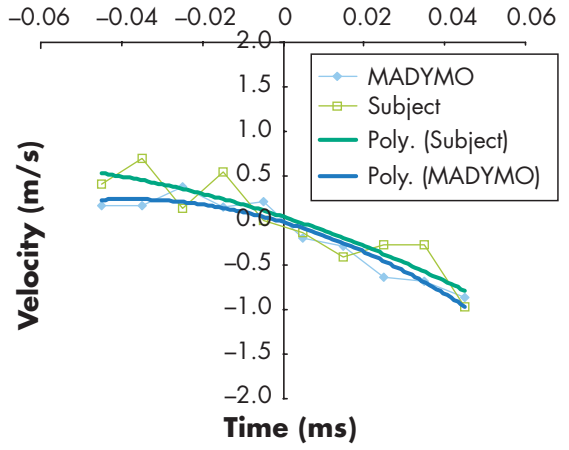

the system energies are similar given that internal neck muscle activities are also similar. A velocity difference of less than 10\% was observed at the time of ball impact confirming satisfactory representation of the test subject (fig 5).

\section{Head kinetic validation}

During the initial subject trials, linear head accelerations were measured via accelerometers mounted to an intraoral device. ${ }^{8}$ The results of the measurements were directly compared with a simulated accelerometer at the same location on the model. A computed peak acceleration of $116 \mathrm{~m} / \mathrm{s}^{2}$ compared well with the mean subject data of $136 \mathrm{~m} / \mathrm{s}^{2}$ (95\% CI 118 to $\left.154 \mathrm{~m} / \mathrm{s}^{2}\right)$. A comparison between the model and subject data is depicted in fig 6. The model data for the head's centre of gravity acceleration are also provided for reference in fig 7 with the $x$ axis corresponding to the fore-aft direction and the $\mathrm{z}$ axis to the inferior-superior direction.

Validation of the neck loads from the numerical model was not possible because comparable data were not readily

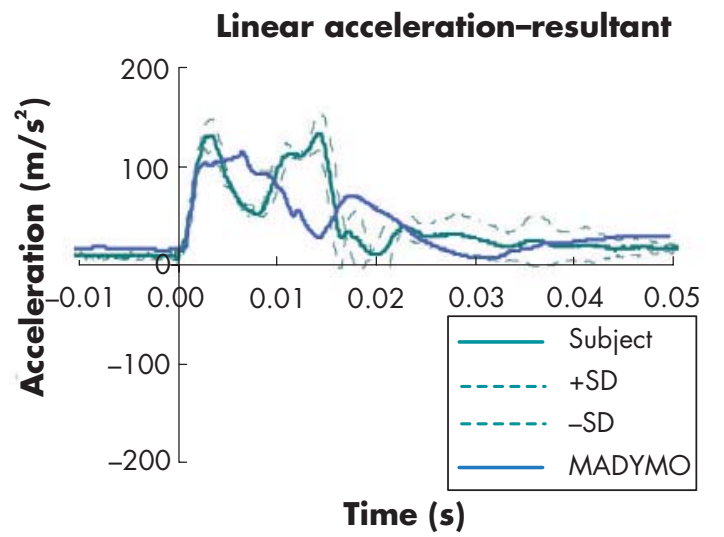

Figure 6 Comparison of resultant head accelerations at the bite plate. available from the test subjects. Alternatively, the neck load time histories of the model were evaluated to assess whether the phasing of the muscle force-time history was reasonable and that the magnitudes of the maximum loads were well below suggested injurious levels for humans. ${ }^{11}$ This was confirmed by the lack of injury in the test subjects.

\section{Parametric study}

Responses from the numerical model are with respect to the local anatomical coordinate system of the body segments as illustrated in fig 8 . Head accelerations $(a, \alpha)$ are taken about the centre of gravity and the neck loads $\left(F_{X}\right.$ shear, $F_{z}$ axial $)$, are taken at the occipital condyle (OC). Torso accelerations originate at the posterior-superior aspect of the Tl vertebra. The results from the numerical model are presented in table 2 as absolute responses and in table 3 relative to the baseline heading scenario. It can be noted that all values of head and neck responses are well below the limits typically associated with acute injury. ${ }^{11}$

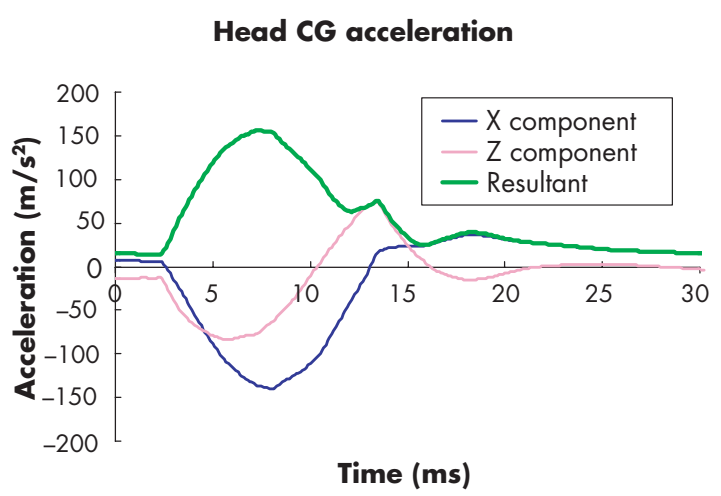

Figure 7 Typical head responses of the numerical model. CG, centre of gravity. 


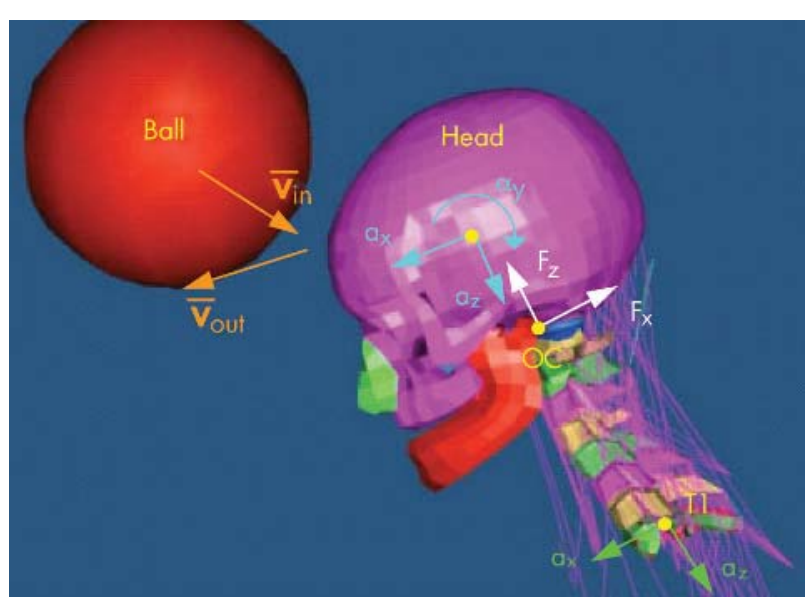

Figure 8 Positive sign conventions of the numerical model for the head, neck, and torso.

\section{DISCUSSION}

With increases of the neck muscle activation levels, peak linear accelerations at the centre of gravity of the head decreased suggesting that better coupling between the head mass and supporting structure was attained. A maximum decrease of $7 \%$ was realised, but it remains to be confirmed whether such tensing can be maintained by a player while still achieving proper heading performance. The peak angular accelerations of the head increased dramatically with greater muscle activity. An increase of up to $48 \%$ was computed and may be attributed to the stiffer head/neck structure causing the head to rebound in a shorter period of time from the ball impact. The differences can be compared with a soft spring which deforms over a longer period of time at a lower resistive force than for a stiffer spring which deforms quickly with a higher force. When considering the combined effects of linear and angular terms with HIP, a net increase in head response is observed. In short, minor gains are seen in terms of linear accelerations but greater losses are found with angular accelerations and the HIP.

The effect of muscle activation on neck axial compression forces and fore-aft shear forces was dramatic. In all cases of increased muscle activity, an increase in axial and shear loads was seen. This can be directly contributed to the muscles preloading the neck structure in addition to the impact force. The effect of head-neck-torso alignment was compared with the baseline response. Overall decreases in head response were observed for peak linear and angular accelerations with a maximum decrease of $11 \%$. The HIP decreases were considerably larger $(-36 \%)$ and were due to the lower magnitudes of the acceleration and duration over which these act thereby resulting in smaller head velocity changes.

The neck axial compression loads increase with headneck-torso alignment which was due to the change in the muscle's line of action compared to the baseline case. Neck shear forces were similarly increased. The ball input velocity was $6.31 \mathrm{~m} / \mathrm{s}$ with a resulting rebound velocity varying from $10.3 \mathrm{~m} / \mathrm{s}$ to $10.7 \mathrm{~m} / \mathrm{s}$ for the muscle tensing case and $9.8 \mathrm{~m} / \mathrm{s}$ for the head-torso alignment case. In all simulations the head impact velocity with the ball was similar $(2.8-3.0 \mathrm{~m} / \mathrm{s})$. The torso accelerations measured at $\mathrm{Tl}$ did not vary greatly with tensed neck muscles having absolute values of 27.8$30.6 \mathrm{~m} / \mathrm{s}^{2}$. The torso alignment scenario resulted in different accelerations of $25.2 \mathrm{~m} / \mathrm{s}^{2}$ due to the different body orientation.

The overall results from the numerical simulations showed that there were benefits with regard to changes in heading technique involving muscle pre-tensing and head-torso
Table 2 Head and neck responses

\begin{tabular}{lllllll}
\hline & $\begin{array}{l}\text { Peak } \\
\text { linear } \\
\text { accel. } \\
\left(\mathbf{m} / \mathbf{s}^{2}\right)\end{array}$ & $\begin{array}{l}\text { Peak } \\
\text { angular } \\
\text { accel. } \\
\left(\mathbf{r a d} / \mathbf{s}^{2}\right)\end{array}$ & $\begin{array}{l}\text { Peak } \\
\text { HIP } \\
(\mathbf{k W})\end{array}$ & $\begin{array}{l}\text { Neck } \\
\text { shear } \\
(\mathbf{N})\end{array}$ & $\begin{array}{l}\text { Neck } \\
\text { axial } \\
(\mathbf{N})\end{array}$ & $\begin{array}{l}\text { Ball exit } \\
\text { velocity } \\
(\mathbf{m} / \mathbf{s})\end{array}$ \\
\hline $\begin{array}{l}\text { Baseline } \\
\begin{array}{l}\text { Muscle at } \\
125 \%\end{array}\end{array}$ & 156 & -374 & 1.44 & 362 & -570 & 10.63 \\
$\begin{array}{l}\text { Muscle at } \\
150 \%\end{array}$ & 155 & -448 & 1.54 & 520 & -927 & 10.33 \\
$\begin{array}{l}\text { Head/torso } \\
\text { aligned }\end{array}$ & 140 & -360 & 0.92 & 388 & -538 & 9.76 \\
\hline Accel., acceleration; HIP, Head Impact Power (index). & & & \\
\hline
\end{tabular}

alignment. In both cases, decreases were seen in head linear acceleration response but were not consistent with the benefit or detriment in either angular acceleration or HIP responses. The inconsistencies indicate that the response parameters must be weighted to establish their relative importance or that a change in heading technique can result in varying benefits. This also suggests that individual techniques are required to optimise a particular response such as HIP or neck loads.

Validation of the model could only be accomplished using a single subject to determine the biofidelity and validity of the predictions. Modelling the population of football players would require validation across the range of subject anthropometry and morphology. A more comprehensive approach incorporating stochastic methods could be employed to represent the effects of heading techniques and environment for all age groups of varying anthropometry and morphology, as well as for varying ball characteristics and impact speeds.

\section{SUMMARY AND CONCLUSION}

Our results show that numerical modelling can re-create the kinematics and kinetics of heading in football based on movements captured in subject trials. In addition, the head and neck response from the model provided values that were realistic in magnitude and duration, allowing the model to function as a biomechanical research tool. Provided the model was used within the validated range of impact scenarios-that is, simple forward heading technique, one may investigate the effects of ball properties, muscle activation, and body alignment on head biomechanics in an effort to introduce strategies for reducing ball impact severity.

While neck muscle activation levels were set through an iterative process to matched observed kinematics, increasing activation levels of the muscle groups would effectively increase coupling between the head and torso. Providing that the three muscle groups represented in the model could be dynamically balanced without modifying the overall heading movement, the effects of coupling could be evaluated at

Table 3 Comparative differences of the numerical model results with heading modifications

\begin{tabular}{|c|c|c|c|c|c|c|}
\hline Scenario & $\begin{array}{l}\text { Peak } \\
\text { linear } \\
\text { accel. } \\
(\%)\end{array}$ & $\begin{array}{l}\text { Peak } \\
\text { angular } \\
\text { accel. } \\
(\%)\end{array}$ & $\begin{array}{l}\text { Peak } \\
\text { HIP } \\
(\%)\end{array}$ & $\begin{array}{l}\text { Neck } \\
\text { shear } \\
(\%)\end{array}$ & $\begin{array}{l}\text { Neck } \\
\text { axial } \\
(\%)\end{array}$ & $\begin{array}{l}\text { Ball exit } \\
\text { velocity } \\
\text { ratio } \\
(\%)\end{array}$ \\
\hline Baseline & 0 & 0 & 0 & 0 & 0 & 0 \\
\hline $\begin{array}{l}\text { Muscle } \\
\text { at } 125 \%\end{array}$ & -1 & 20 & 7 & 44 & 63 & -3 \\
\hline $\begin{array}{l}\text { Muscle } \\
\text { at } 150 \%\end{array}$ & -7 & 48 & 6 & 79 & 119 & 0 \\
\hline $\begin{array}{l}\text { Head/torso } \\
\text { aligned }\end{array}$ & -11 & -4 & -36 & 7 & -6 & -8 \\
\hline
\end{tabular}




\section{What is already known on this topic}

- Heading is an important aspect of the game of football but the biomechanical understanding is limited.

- Numerical models can provide insight into the biomechanical effects of head impacts.

- Passive models have been implemented under a range of ball impact conditions but have not addressed impact reduction through modified heading techniques and investigation of ball properties.

- Limited validation and simplified implementation of some models are areas of concern.

muscle activation levels representing neck muscle development of different athletes. Finally, variations observed in body alignment - that is, the relative position of the head, cervical spine, and torso, could be studied to investigate the notion of coupling between the body segments.

Our model is limited in that the kinematics were matched to a single heading scenario and subject, and to the adult size of the model. The results for other circumstances are not known. Further exploration of the techniques with human subjects under varying heading types and ball impact speeds would confirm the observed merits.

The MADYMO code does provide a 5 th percentile female human model that would represent the stature of a smaller athlete but the detailed head and neck are not available at this time. Monte Carlo simulations are another way in which a range of head accelerations or neck loads could be determined for various sized adults and youth players. ${ }^{12} \mathrm{~A}$ stochastic software module for use with MADYMO was released after the completion of this study, and it could be used to investigate simple parameters such as ball mass/ pressure or impact speed. To study the effect of player size, a scalable model or series of models would be required. Finite element methods applied to both the head and ball could provide insight into the contact event which would be useful with respect to football development or brain injury potential if an anatomical head model was implemented. Loading conditions generated by different heading scenarios such as side to side head motion with temporal impacts are of interest and could be further investigated with the model and protocol developed in this study.

Heading in football is essential to the defensive and offensive strategies of the game. Modification of game rules or heading techniques will remain a remote possibility until a greater understanding of the biomechanics and injury potential are obtained. The biomechanical modelling techniques presented in this paper can help provide insight into head impact response and influence of heading technique. When combined with subject and epidemiological data, effective preventive measures can be formulated and the benefits realised.

\section{ACKNOWLEDGEMENTS}

This research has been supported through the contributions of the FIFA Medical Assessment and Research Centre (F-MARC). The
What this study adds

- An advanced numerical model with detailed head and neck was validated and implemented for active heading of the ball.

- The model was used to investigate potential strategies for head impact severity reduction through modified heading techniques and ball characteristics.

- Initial recommendations for heading techniques are provided to help reduce head impact severity.

- Further research with the model will gain more comprehensive understanding of heading biomechanics.

authors wish to acknowledge the participation of the football teams, the Gloucester Hornets and Kanata Soccer (Ontario, Canada) for participation of their players as test subjects.

\section{Authors' affiliations}

N Shewchenko, C Withnall, M Keown, R Gittens, Biokinetics and Associates Ltd., Ontario, Canada

J Dvorak, FIFA Medical Assessment and Research Centre, Zurich, Switzerland

Competing interests: none declared

\section{REFERENCES}

1 Schneider K, Zernicke R. Computer simulation of head impact: estimation of head-injury risk during soccer heading. Int J Sport Biomech 1988:4:258-371.

2 Ziejewski M, Yliniemi E, Ramaswamy S. Modeling of head/neck response to low severity head impacts. ATB Users Group Conference. Wichita, KS: National Institute of Aviation Research, 2000.

3 Ziejewski M, Swenson R, Schanfield P, et al. A biomechanical examination of the efficacy of soccer protective headgear in reducing trauma to the head from low impacts. Brain Injury Association 20th Annual Symposium, Atlanta, GA, 2003.

4 Queen RM, Weinhold PS, Kirkendall D, et al. Theoretical study of the effect of ball properties on impact force in soccer heading. Med Sci Sports Exerc 2003;35:2069-76

5 Beusenberg M, Shewchenko N, Newman J. Head, neck and body coupling in reconstructions of helmeted head impacts. International Research Council on the Biomechanics of Impact, Isle of Man, UK, 2001:295-310.

6 van der Horst M. Human head neck response in frontal, lateral and rear end impact loading: modelling and validation. Eindhoven: Technische Universiteit Eindhoven, 2002.

7 Meijer R, Ono K, Van hoof J, et al. Simulation of human neck response to direct loading of the head. Society of Automotive Engineers, Spring Convention, 2002:280-3.

8 Shewchenko N, Withnall C, Keown M, et al. Heading in football. Part 1: Development of biomechanical methods to investigate head response. Br J Sports Med 2005;39(suppl I):i10-i25.

9 Shewchenko N, Withnall C, Keown M, et al. Heading in football. Part 3: Effect of ball properties and head response. Br J Sports Med 2005;39(suppl I):i33-i39.

10 Newman J, Shewchenko N, Welbourne E. A proposed new biomechanical head injury index assessment function - the maximum power index. 44th Stapp Car Crash Conference, SAE International, 6-8 November 2000, Atlanta, GA.

11 Mertz H, Prasad P, Irwin AL. Injury risk curves for children and adults in frontal and rear collisions. 41 st Stapp Car Crash Conference, SAE International, 1330 November 1997, Lake Buena Vista, FL.

12 Babbs CF. Biomechanics of heading a soccer ball: implications for player safety. West Lafayette, IN: Purdue University, 8 August, 2001. 